\title{
OSCILLATTORY FLOW OF CASSON FLUID BETWEEN PARALLEL PLATES WITH AN INCLINED MAGNETIC FIELD
}

\author{
Rafiuddin $^{1}$ and Noushima Ghouri ${ }^{2}$ \\ ${ }^{1}$ Department of Humanities and Applied Science,CVR College \\ of Engg and Tech,Telagana,India \\ ${ }^{2}$ Department of Mathematics,, College of Science and \\ Arts, Taibah University,KSA \\ ORCID ID : 0000-0003-4575-6066
}

\begin{abstract}
The study of heat and mass transfer of oscillatory casson flow in porous medium subject to an inclined magnetic field, radiative heat flux and heat source is presented. It is supposed that Casson fluid is little conductive and produced emf is insignificant. The solutions of coupled partial differential equations of velocity, ,temperature and concentration profiles are found using Galerkins technique of finite element method. The effect of various parameters such as Reynolds number Re, Grashoff number Gr, Solute Grashoff number Gc, Peclet number Pe, Hartman number Ha, Scmidth number Sc, Permeability parameter K, Radiative parameter R, Heat generation parameter $\mathrm{S}$, Chemical reaction parameter $\mathrm{Kr}$ and frequency parameter $\omega$ on velocity, temperature and concentration are shown graphically and skin friction, Nusselts number and Sherwood number are discussed by tables
\end{abstract}

Keywords: Free convective,Skin-friction,Nusselt number,Sherwood number,Casson fluid.

\footnotetext{
${ }^{*}$ Corresponding author email:noushimahg@yahoo.com
} 


\section{Introduction}

Fluid flow with heat and mass transfer in porous medium has many applications on geophysics, petro chemical engineering, oceanography, boundary layer control in aerodynamics and polymer technology. Magneto hydrodynamic flows of electrically conductive fluid plays important role in MHD bearings, power generators, metrology, astrophysics to study the stellar and solar structures, inter stellar matters and radio wave propagation through ionosphere, extraction, electromagnetic propulsion and has industrial applications in molten iron flow, extraction of crude oil, electrostatic precipitation. [1] presented heat transfer in MHD flow between parallel finitely conductive plates in presence of a transverse magnetic field.[2] considered the steady flow of a viscous incompressible fluid bounded by two infinite insulated horizontal plates and heat transfer through it examined.[4] probed the boundary layer memory flow and given analytic solution for heat and mass transfer considering hall current effects. [5] discussed a MHD flow of a viscous fluid between two parallel porous plates. The effect of magnetic field and pressure gradient on velocity and temperature focused.[6]analyzed the MHD flow through parallel plates with heat and mass transfer and showed that Hartman number reduces the flow.[7] ascertained that Hatmann number enhances induced magnetic field and magnetic Reynolds number influenced velocity in their study of heat and mass transfer between vertical parallel plate.[8] investigated MHD Poiseulle flow between two parallel plates with heat transfer an showed that Hartmann number suppresses flow and Prandtle number subsides temperature profles. [9] discussed the steady Poiseuille flow between two infinite parallel plates in an inclined magnetic field and ascertained magnetic field reduces the velocity.[11] obtained the analytic solutions for velocity,temperature,concentration, skinfriction, heat and mass transfer using Laplace technique.[12] studied MHD free convective non-newtonian flow with variable permeability. Numerical solution of unsteady two dimensional hydromagnetic flow with heat and mass transfer of casson fluid discussed by [13].[14] given observation of unsteady MHD poiseuille flow between two infinite parallel plates with heat and mass transfer subjected to porous material and an inclined magnetic field.[15]discussed heat and mass transport on MHD free convective flow through porous medium past an infinite vertical plate.[16]shown the effect of an inclined magnetic field is to reduce flow of dusty fluid in unsteady case.[17] discussed effects of variable thermal conductivity and heat source/sink on MHD flow near a stagnation point on a boundary conditions.[18] highlighted the heat and mass transfer in steady flow in presence of an inclined magnetic field and found velocity first decreases and then rises gradually. 
The aim of the paper is to extend the work of [10] to memory fluid with mass transfer

\section{Formulation of the problem}

Consider the oscillatory casson fluid flow of an electrically conducting fluid and heat transfer between infinite parallel plates at a distance $d$ apart filled with a porous medium under the influence of an inclined magnetic field and heat source. The $x^{*}$-axis is taken along the plate when $\left(y^{*}=0\right)$ and the $y^{*}$-axis is taken normal to the plate.As the plates are infinite in length ,the velocity and temperature fields are functions of $y^{*}$ and $t^{*}$ only.

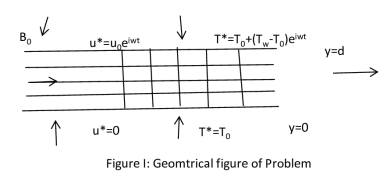

Figure 1: Geometrical representation of problem

Under the above assumption and usual Boussineq approximation ,the governing equations of motion,energy and concentration are as follows

$$
\begin{gathered}
\frac{\partial u^{*}}{\partial t^{*}}=-\frac{1}{\rho} \frac{\partial p^{*}}{\partial x^{*}}+\nu \beta^{*} \frac{\partial^{2} u^{*}}{\partial y^{* 2}}+g \beta\left(T^{*}-T_{0}\right)+g \beta^{* *}\left(C^{*}-C_{0}\right)-\frac{\sigma_{e} B_{0}^{2} \sin ^{2} \phi}{\rho} u^{*}-\frac{\nu}{K^{*}} u^{*} \\
\frac{\partial T^{*}}{\partial t^{*}}=\frac{\kappa}{\rho C_{p}} \frac{\partial^{2} T^{*}}{\partial y^{* 2}}-\frac{1}{\rho C_{p}} \frac{\partial q_{r}}{\partial y^{*}}+\frac{Q_{0}}{\rho C_{p}}\left(T^{*}-T_{0}\right) \\
\frac{\partial C^{*}}{\partial t^{*}}=D \frac{\partial^{2} C^{*}}{\partial y^{* 2}}-K_{r}\left(C^{*}-C_{0}\right)
\end{gathered}
$$

The boundary conditions are given by:

$$
\begin{gathered}
y^{*}=0: u^{*}=0, T^{*}=T_{0}, C^{*}=C_{0} \\
y^{*}=d: u^{*}=U_{0} e^{i \omega^{*} t^{*}}, T^{*}=T_{0}+\left(T_{w}-T_{0}\right) e^{i \omega^{*} t^{*}}, C^{*}=C_{0}+\left(C_{w}-C_{0}\right) e^{i \omega^{*} t^{*}}
\end{gathered}
$$


It is assumed that the fluid is optically thin with a relative low density and radiative heat flux is according to [3] and given by

$$
\frac{\partial q_{r}}{\partial y^{*}}=4 \alpha^{2}\left(T^{*}-T_{0}\right)
$$

Introducing the following dimensionless quantities,

$u=\frac{u^{*}}{U_{0}}, x=\frac{x^{*}}{d}, y=\frac{y^{*}}{d}, t=\frac{U_{0}}{d} t^{*}, \omega=\frac{d}{U_{0}} \omega^{*}, P=\frac{d}{\nu \rho U_{0}} P^{*}, \theta=\frac{T^{*}-T_{0}}{T_{w}-T_{0}}, R e=\frac{U_{0} d}{\nu}$

$H a^{2}=\frac{\sigma_{e} B_{0}^{2} d^{2}}{\nu \rho}, G r=\frac{g \beta d^{2}\left(T_{w}-T_{0}\right)}{\nu U_{0}}, K^{2}=\frac{d^{2}}{K^{*}}, P e=\frac{U_{0} d \rho C_{p}}{\kappa} R^{2}=\frac{4 \alpha^{2} d^{2}}{\nu \rho C_{p}}$,

$S=\frac{Q_{0} d^{2}}{\kappa}, S_{c}=\frac{D}{\nu}, K_{r}^{*}=\frac{k_{r} d}{u_{0}}, G c=\frac{g \beta_{1} d^{2}\left(C_{w}-C_{0}\right)}{\nu u_{0}}, \phi=\frac{C-C_{0}}{C_{w}-C_{0}}$,

into eq (2.1),(2.2) and (2.3) we get

$$
\begin{gathered}
R e \frac{\partial u}{\partial t}=-\frac{\partial p}{\partial x}+\beta^{*} \frac{\partial^{2} u}{\partial y^{2}}+G r \theta-\left(M^{2}+K^{2}\right) u+G c \phi \\
P e \frac{\partial \theta}{\partial t}=\frac{\partial^{2} \theta}{\partial y^{2}}-R^{2} \theta+S \theta \\
\frac{\partial \phi}{\partial t}=\frac{1}{S c} \frac{\partial^{2} \phi}{\partial y^{2}}-K_{r} \phi
\end{gathered}
$$

where $M=H a \sin \psi$

The boundary conditions are reduced to

$$
\begin{gathered}
y=0: u=0, \theta=0, \phi=0 \\
y=1: u=e^{i \omega t}, \theta=e^{i \omega t}, \phi=e^{i \omega t}
\end{gathered}
$$

\section{Method of Solution}

For a purely oscillatory flow,substituting

$$
-\frac{\partial P}{\partial x}=\lambda e^{i \omega t}, u(y, t)=u_{0}(y) e^{i \omega t}, \theta(y, t)=\theta_{0}(y) e^{i \omega t} a n d \phi(y, t)=\phi_{0}(y) e^{i \omega t}
$$

into equations $(2.7),(2.8)$ and(2.9)we get

$$
\begin{gathered}
u_{\theta}^{11}-v_{1} u_{0}=-\frac{1}{\beta^{*}}\left(\lambda+G r \theta_{0}+G c \phi_{0}\right) \\
\theta_{0}^{11}-\nu_{2} \theta_{0}=0
\end{gathered}
$$




$$
\phi_{0}^{11}-v_{3} \phi_{0}=0
$$

Now,the corresponding boundary conditions are

$$
\begin{aligned}
& y=0: u_{0}=0, \theta_{0}=0, \phi_{0}=0 \\
& y=1: u_{0}=1, \theta_{0}=1, \phi_{0}=1
\end{aligned}
$$

Equations (3.2), (3.3)and(3.4)are ordinary second order coupled differential equations and are solved under the boundary conditions(3.5) and (3.6).Through straight forward calculations $u_{0}$ and $\theta_{0}, \phi_{0}$ are found.Finally,the expressions of $\mathrm{u}(\mathrm{y}, \mathrm{t}), \theta(y, t)$ and $\phi(y, t)$ are known as given below

$$
\begin{gathered}
u_{0}=l_{5}\left(y^{2}-y\right)+y+i l_{6}\left(y^{2}-y\right) \\
u(y, t)=u_{0} e^{i \omega t} \\
\theta_{0}=\left(m_{1}+1\right) \cos \omega t-m_{2} \sin \omega t \\
\theta(y, t)=\theta_{0} e^{i \omega t} \\
\phi_{0}=\left[n_{1} y^{2}+\left(1-n_{1}\right) y\right] \cos \omega t-n_{2}\left(y^{2}-y\right) \sin \omega t \\
\phi(y, t)=\phi_{0} e^{i \omega t}
\end{gathered}
$$

The dimensionless stress tensor in terms of the skin-friction coefficient at upper the plates are given by

$$
C_{f}=\left(l_{5}+1\right) \cos \omega t-l_{6} \sin \omega t
$$

The non dimensional rate of heat transfer in terms of the Nusselts number at the upper plate is given by

$$
N u=\left(m_{1}+1\right) \cos \omega t-m_{2} \sin \omega t
$$

The non dimensional rate of mass transfer in terms of Sherwood number at the upper plate is given by

$$
S h=\left(n_{1}+1\right) \cos \omega t-n_{2} \sin \omega t
$$

Constants $m_{1}, m_{2}, n_{1}, n_{2}, l_{1} \ldots$ are not presented for the sake of brevity. 


\section{Results and Conclusion}

The effect of an inclined magnetic field,radiation and heat source on an oscillatory flow of an incompressible Casson fluid through a porous medium between parallel plates are investigated.Equations of momentum, energy and concentration which governs the fluid flow, heat transfer and mass transfer are solved.The effects of various physical parameters on the fluid velocity, temperature and concentration are depicted graphically and skin friction, Nusselt number and Sherwood number at the upper plate are discussed numerically. Table 1 highlights that,Skin friction $C_{f}$ dominates with rise in Hartmann number Ha,phase angle $\psi$,permeability parameter K,frequency $\omega$,radiation parameter R,Schimdt number Sc and chemical reaction parameter Kr.And also,skin friction reduces with the rise in Grashoff number Gr,solute Grashoff number Gc,Reynolds number Re,Peclet number Pea and heat source parameter S.

\begin{tabular}{|c|c|c|c|c|c|c|c|c|c|c|c|c|c|c|c|}
\hline$\alpha$ & $\mathrm{Ha}$ & $\lambda$ & $\mathrm{Re}$ & K & $\mathrm{R}$ & $\mathrm{S}$ & $\omega$ & $\mathrm{Kr}$ & $\mathrm{Gr}$ & Gc & $\mathrm{Pe}$ & $\mathrm{Sc}$ & $\mathrm{t}$ & $\psi$ & $\mathrm{Cf}$ \\
\hline 0.25 & 2 & 0.1 & 2 & 1 & 2 & 5 & 1 & 1 & 10 & 1 & 0.71 & 2 & 0 & $\frac{\pi}{4}$ & -1.440590 \\
\hline 0.25 & 4 & 0.1 & 2 & 1 & 2 & 5 & 1 & 1 & 10 & 1 & 0.71 & 2 & 0 & $\frac{I}{4}$ & 0.540196 \\
\hline 0.25 & 2 & 0.2 & 2 & 1 & 2 & 5 & 1 & 1 & 10 & 1 & 0.71 & 2 & 0 & $\frac{11}{4}$ & -1.44414 \\
\hline 0.25 & 2 & 0.1 & 4 & 1 & 2 & 5 & 1 & 1 & 10 & 1 & 0.71 & 2 & 0 & $\frac{\pi}{4}$ & -0.382325 \\
\hline 0.25 & 2 & 0.1 & 2 & 2 & 2 & 5 & 1 & 1 & 10 & 1 & 0.71 & 2 & 0 & $\frac{11}{4}$ & -0.425023 \\
\hline 0.25 & 2 & 0.1 & 2 & 1 & 4 & 5 & 1 & 1 & 10 & 1 & 0.71 & 2 & 0 & $\frac{11}{4}$ & 0.460438 \\
\hline 0.25 & 2 & 0.1 & 2 & 1 & 2 & 6 & 1 & 1 & 10 & 1 & 0.71 & 2 & 0 & $\frac{11}{4}$ & -1.857449 \\
\hline 0.25 & 2 & 0.1 & 2 & 1 & 2 & 5 & 2 & 1 & 10 & 1 & 0.71 & 2 & 0 & $\frac{\pi}{4}$ & -0.436621 \\
\hline 0.25 & 2 & 0.1 & 2 & 1 & 2 & 5 & 1 & 2 & 10 & 1 & 0.71 & 2 & 0 & $\frac{I^{4}}{4}$ & -1.405197 \\
\hline 0.25 & 2 & 0.1 & 2 & 1 & 2 & 5 & 1 & 1 & 15 & 1 & 0.71 & 2 & 0 & $\frac{11}{4}$ & -3.096498 \\
\hline 0.25 & 2 & 0.1 & 2 & 1 & 2 & 5 & 1 & 1 & 10 & 2 & 0.71 & 2 & 0 & $\frac{\pi}{4}$ & -1.687013 \\
\hline 0.25 & 2 & 0.1 & 2 & 1 & 2 & 5 & 1 & 1 & 10 & 1 & 1.0 & 2 & 0 & $\frac{11}{4}$ & -1.446566 \\
\hline 0.25 & 2 & 0.1 & 2 & 1 & 2 & 5 & 1 & 1 & 10 & 1 & 0.71 & 4 & 0 & $\frac{11}{4}$ & -1.404351 \\
\hline 0.25 & 2 & 0.1 & 2 & 1 & 2 & 5 & 1 & 1 & 10 & 1 & 0.71 & 2 & 1 & $\frac{11}{4}$ & 0.543622 \\
\hline 0.25 & 2 & 0.1 & 2 & 1 & 2 & 5 & 1 & 1 & 10 & 1 & 0.71 & 2 & 0 & $\frac{11}{3}$ & -0.954435 \\
\hline 0.5 & 2 & 0.1 & 2 & 1 & 2 & 5 & 1 & 1 & 10 & 1 & 0.71 & 2 & 0 & $\frac{\Pi 1}{4}$ & -1.440590 \\
\hline
\end{tabular}

Table 1: Numerical Values of skin friction coefficient at the upper plate for the various values of physical properties 
Table 2 shows ,the rate of heat transfer i:e Nusselt number Nu increases with increase in radiation parameter $\mathrm{R}$ and frequency $\omega$.And decreases with increase in heat source parameter $\mathrm{S}$ and time t.

\begin{tabular}{|c|c|c|c|c|c|}
\hline $\mathrm{R}$ & $\mathrm{S}$ & $\mathrm{Pe}$ & $\omega$ & $\mathrm{t}$ & $\mathrm{Nu}$ \\
\hline 2 & 5 & 0.71 & 1 & 0 & 0.582642 \\
\hline 4 & 5 & 0.71 & 1 & 0 & 1.113269 \\
\hline 2 & 6 & 0.71 & 1 & 0 & 0.423934 \\
\hline 2 & 5 & 1.0 & 1 & 0 & 0.588231 \\
\hline 2 & 5 & 0.71 & 2 & 0 & 0.599597 \\
\hline 2 & 5 & 0.71 & 1 & 1 & 0.226686 \\
\hline
\end{tabular}

Table 2: Numerical Values of rate of heat transfer i:e Nusselt number at the upper plate for the various values of physical properties

From Table 3 we can conclude that ,the Schimdt number Sc,chemical reaction parameter $\mathrm{Kr}$,frequency $\omega$ influences the mass transfer .And also, with time t mass transfer dips.

\begin{tabular}{|c|c|c|c|c|}
\hline Sc & Kr & $\omega$ & t & Sh \\
\hline 2 & 1 & 1 & 0 & 1.43189 \\
\hline 4 & 1 & 1 & 0 & 1.707547 \\
\hline 2 & 2 & 1 & 0 & 1.72500 \\
\hline 2 & 1 & 2 & 0 & 1.50000 \\
\hline 2 & 1 & 1 & 1 & 0.66385 \\
\hline
\end{tabular}

Table 3: Numerical Values of rate of mass transfer i:e Sherwood number at the upper plate for the various values of physical properties

From Figures 1-7 it is observed that, velocity shoots up for higher values of Grashoff number Gr,Solute Grashoff number Gc,heat generation parameter S .whereas, velocity has reverse effect for low values of Hartmann number Ha,Reynolds number Re,Radiation parameter R,frequency $\omega$,permeability parameter K. Peclet number Pe and chemical reaction parameter Kr has no affects on velocity. 

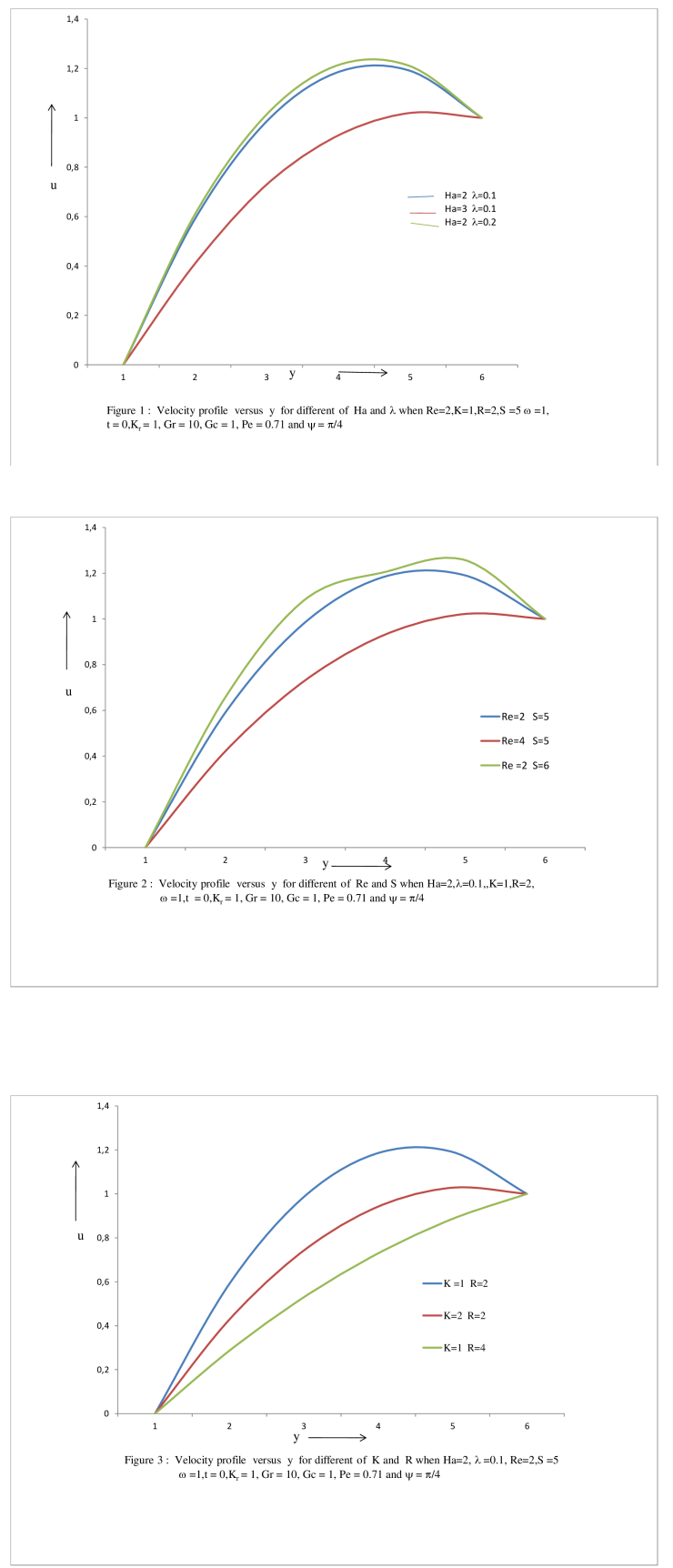

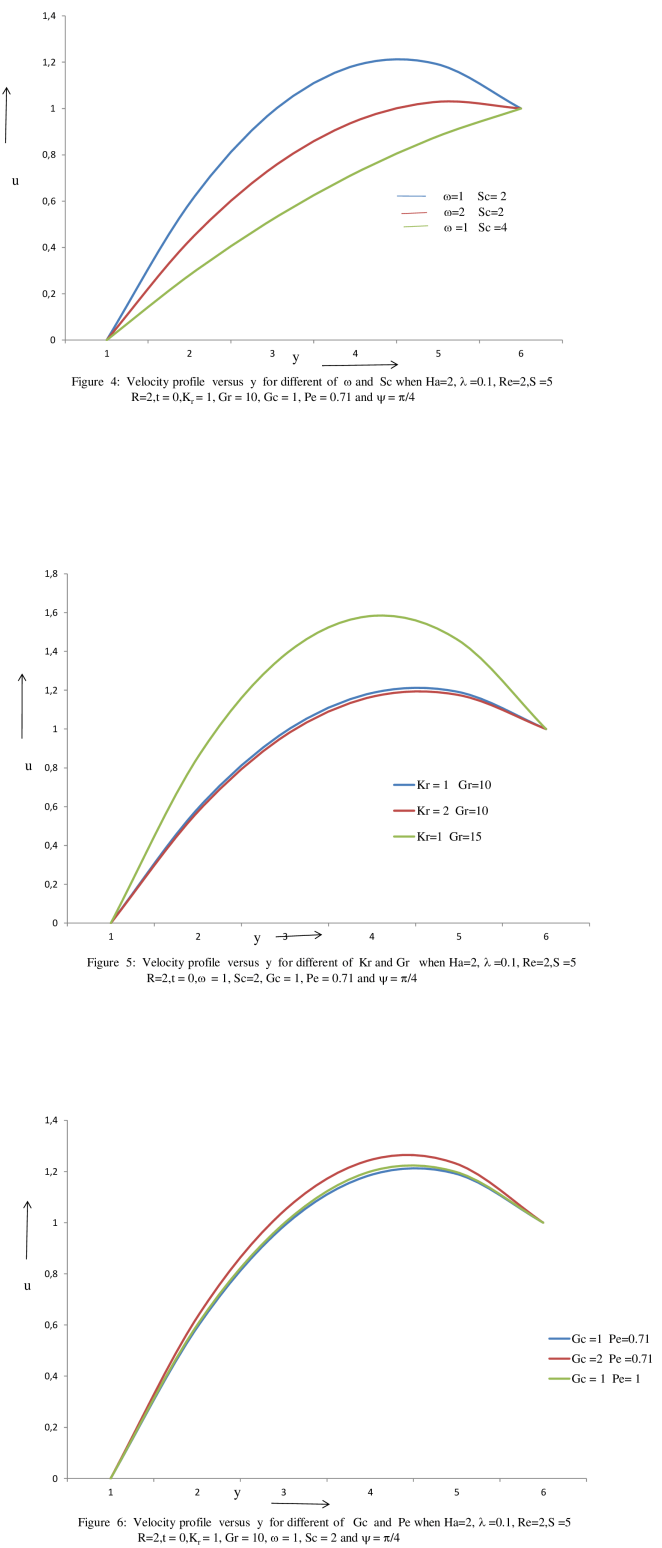


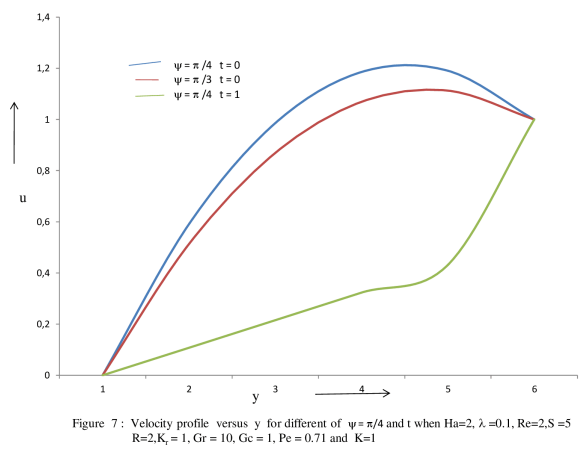

Figures 8-10 depicts that,Heat generation parameter $\mathrm{S}$ influences the temperature $\theta$ whereas Radiation parameter R,Peclet number Pe ,frequency $\omega$ and t time suppresses .
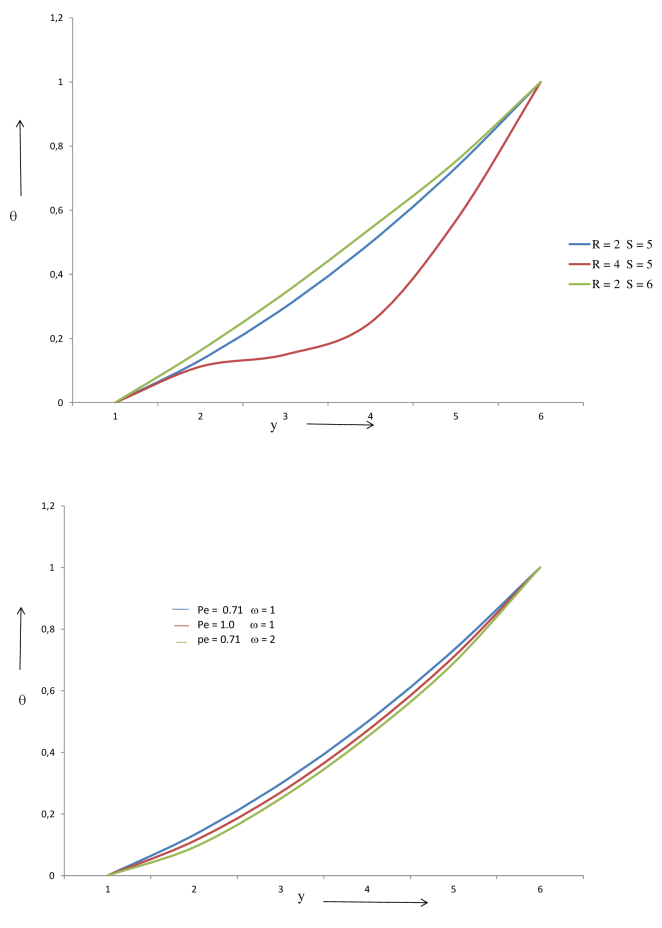


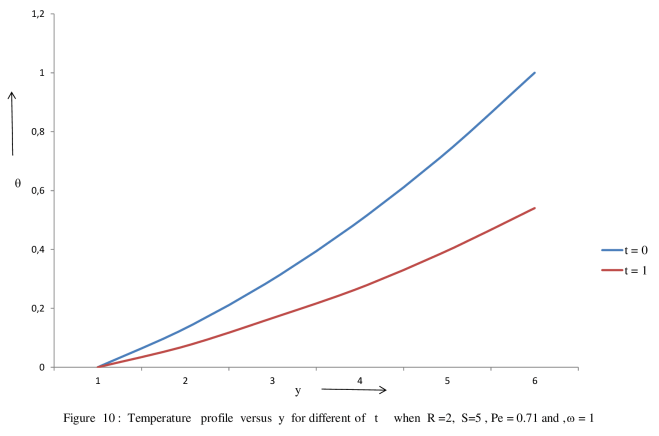

Figures 11-12 shows that,Concentration $\phi$ reduces for higher values of Schimdt number Sc,chemical reaction parameter $\mathrm{Kr}$ and frequency $\omega$.
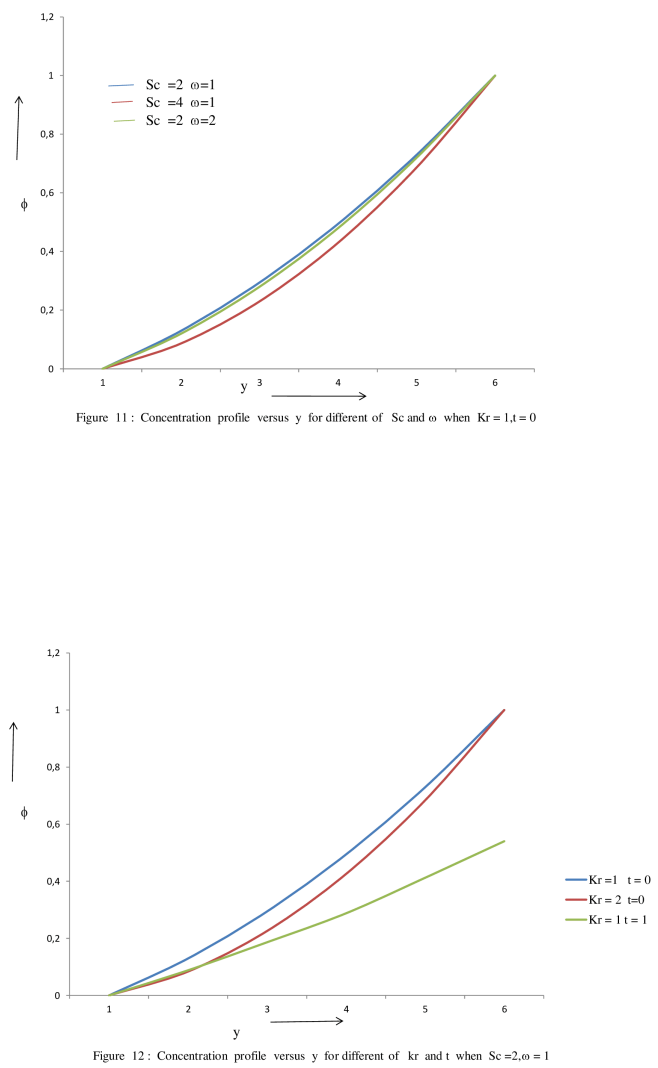
From Figure 13, we draw conclusion that velocity decreases with increase in Casson parameter.

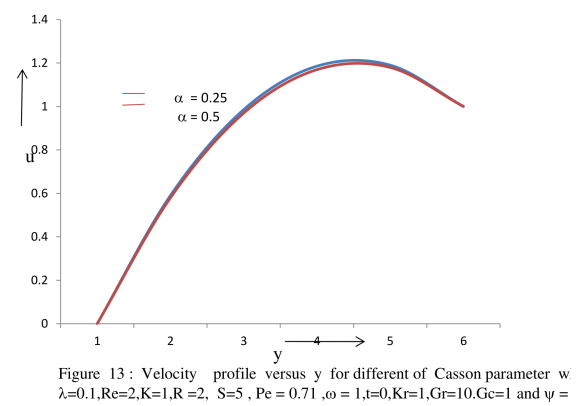

\section{Nomenclature:}

$B_{0}$ Strength of the magnetic field

$C_{p}$ specific heat at constant pressure

$P_{e}$ Peclet number

Gr Grashoff number

$Q_{0}$ heat generation/absorption constant

Gc Solute Grashoff number

g acceleration due to gravity

qr radiative heat flux in $y^{*}$

Ha Hartmann Number

$S$ heat source parameter

K permeability parameter

$K^{*}$ permeability of porous medium

$\mathrm{P}$ dimensionless pressure

$T^{*}$ fluid temperature

$T_{0}$ Temperature of plate at $y^{*}=0$

$T_{w}$ Temperature of plate at $y^{*}=\mathrm{d}$

$C_{0}$ Concentration of mass of plate at $y^{*}=0$ 
$\alpha$ mean radiation absorption coefficient

$R$ radiative parameter in the $y^{*}$ direction

$\beta$ coefficient of the thermal expansion

$R_{e}$ Reynolds number

$\beta$ Coefficient of the thermal expansion

$\theta$ dimensonless temperature

$t$ dimensionless time

$\kappa$ thermal conductivity

$t^{*}$ time

$u$ dimensionless velocity along $\mathrm{x}$ axis

$\nu$ kinematic viscosity

$\sigma_{e}$ Electrical conductivity

$u^{*}$ fluid velocity in $x^{*}$ direction

$y$ dimensionless coordinate axis

$\beta^{* *}$ Coefficient of mass concentration

$\rho$ fluid density

$\lambda$ wave length

$\psi(0 \leqslant \psi \leqslant \pi)$ angle between velocity and strength of the magnetic field

\section{References}

[1] Alpher,A.R(Sept. - 1961):Heat transfer in magneto hydrodynamic flow between parallel plates. -Int.Jour.of heat and mass transfer.vol.3(2).pp.108-112.

[2] Attia,H.A and Kotb,N.A(1996):Acta Mechanica,vol.117,p.215-220.

[3] Cogley A.C,Vincenti W.G and Gill S.E.,(1968):Differential approximation for radiative transfer in a non-gary gas near equilibrium,AIAAJ,vol 6,p.551-553.

[4] Dastagiri Babu,D., Venkateshwarlu,B., and Keshav Reddy,B.,(2018):Int.J.Pure and Appl.Maths,vol 119(15),p.87-103,2018.

[5] Ganesh S. and Krishnabai S.(2006):Int .Jour. of Appl. Engg..research., vol.6, pp.2450-2425.

[6] Hanvey,R.R., Khare,R.K., and Ajit Paul, (2017): IJSIMR, vol.5, (4), pp.18-22.

[7] Hemamalini,P.T., and Shanti,M., (2018):Int.J.Mechanical.Engg and Tech,vol 9(13),p.521-534,2018. 
[8] Idowu,A.S., and Olabode,J.O., (May-June2014): .,(IOSR-JM), vol.10 (3), ver.11 pp.47-53.

[9] Manyonge,W.A., Kiema,D.W., and Iyaya,C.C.W., (2012): . - Int. J. Pure Appl. Mathematics, vol 76 (5), pp.661-668.

[10] Mehta,T., Mehta,R., and Mehta,A.,(2020):Int.J.Applied Mech.Engg,vol 25,No 2,p.88-102,2020.

[11] Nagamanemma,K., Varma,S.V.K., Raju,G.S.S., and Raju.M.C., (2015), Int. Jour. Of.Adv. Information Science And Technology,vol.38 (38), pp.133-146.

[12] Noushima Humera,G., and Rafiuddin (2020):Int.J.Emerging Tech.Adv.Engg,vol 10,issue 5,2020.

[13] Rafiuddin and Noushima Humera,G., (2020): J.Mech anics of continua and Mathematical Sciences,vol 15,No 9,p.17-30,2020.

[14] Rajput,U.S., and Gaurav Kumar (2015):Int.J.Mathematical Archive,vol6(11) ,p.128-134,2015.

[15] Sadiq Basha,P.M., and Nagarathna.N., (2019): -Int. Jour. of Applied Engg.research, vol.14, (21), pp.4067-4076.

[16] Sandeep,N., and Sugunamma,V., (2013): -Int.Jour.of Applied Maths Modelling, Vol.1,(1),pp. 16-33.

[17] Sharma P.R. and Singh G. (2008): Effects of variable thermal conductivity andheat source/sink on MHD flow near a stagnation point on a linearly stretching sheet,J. of Appl. Fluid Mechanics, vol.2, pp.13.

[18] Srivatsava,M., and Khare.R., (2016): IJESRT, vol.5(11), pp.78-82.2016. 